\title{
EL DESAFÍO DE LA CONVIVENCIA INTERCULTURAL
}

\author{
María José Aguilar Idáñez* \\ Daniel Buraschi*
}

\begin{abstract}
La diversidad cultural constituye un asunto central y de importancia cada vez más creciente en la dinámica y evolución de las sociedades actuales. La gestión de este hecho, sin embargo, se realiza desde perspectivas y respuestas muy variadas y, no pocas veces, de dudosa eficacia en el terreno de la convivencia ciudadana para una ciudadanía inclusiva. En este artículo se analizan las tres respuestas más comunes a este desafío, que se han denominado y caracterizado como "asimilación subalterna", "racismo culturalista" y "estética intercultural". Asimismo, se han identificado algunas de las limitaciones, carencias y problemas que estas respuestas evidencian. Frente a estas se destaca la necesidad de desarrollar una respuesta interculturalista crítica y transformadora, que se considera una alternativa válida para el desarrollo de una convivencia basada en el diálogo, la igualdad y el reconocimiento de las diferencias.
\end{abstract}

Palabras-clave: Interculturalidad; Convivencia; Racismo culturalista; Asimilacionismo; Integración.

La gestión de la diversidad cultural representa uno de los mayores retos de nuestras sociedades. La inmigración y la diversidad cultural no son fenómenos coyunturales sino estructurales que necesitan respuestas globales encaminadas a la integración efectiva y a la convivencia intercultural. Esto significa sustituir las tradicionales políticas de

\footnotetext{
* Doctora en Sociología. Catedrática de Trabajo Social de la Universidad de Castilla-La Mancha (España). Directora del Máster online en Inmigración e Interculturalidad y Directora del Grupo de Interdisciplinar de Estudios sobre Migraciones, Interculturalidad y Ciudadanía (GIEMIC). Autora de más de 100 publicaciones científicas y 3 películas documentales sobre migraciones e interculturalidad. Albacete/España.

* Doctor en Ciencias de la Educación por la Universidad de Padua, investigador del GIEMIC, Profesor del Máster sobre Migraciones e Interculturalidad de la UCLM y miembro de la Asociación para la Educación a la Convivencia Mosaico Canarias. Tenerife/España.
} 
intervención, que surgen como respuesta a situaciones de urgencia, por proyectos globales de intervención encaminados a la integración participativa y efectiva, es decir, a la construcción de una sociedad intercultural.

En este artículo proponemos al lector algunas reflexiones críticas sobre los modelos dominantes de gestión de la diversidad con el objetivo de ayudar a conocer y comprender cómo transitar caminos de reflexión y de intervención que nos conduzcan a una sociedad verdaderamente intercultural. Una sociedad de derecho y democrática para todos y todas los que en ella vivimos (no sólo para los que han nacido en determinado lugar o continente).

\section{El multiculturalismo como hecho}

Multiculturalismo es un término polisémico: por un lado es una etiqueta que se utiliza para describir un hecho social, la coexistencia en un mismo espacio social de personas con diferentes horizontes culturales; por el otro es un concepto que hace referencia a un proyecto político para la gestión de la diversidad cultural que se ha desarrollado en las últimas décadas en Canadá, Estados Unidos e Inglaterra.

Respecto al significado descriptivo del término multiculturalismo hay que hacer tres observaciones importantes. La primera es que España ha sido siempre una sociedad multicultural, en el sentido que en nuestro territorio coexistimos desde hace mucho tiempo personas y grupos con diferentes lenguas, de diferentes etnias (pensemos en la minoría gitana, por ejemplo), diversas nacionalidades (pensemos en los jubilados alemanes y británicos que desde hace décadas viven en las zonas costeras), diferentes religiones, diversas tradiciones, trayectorias históricas y culturales; y, por tanto, podemos constatar fácilmente una importante diversidad cultural interna. Sin embargo, no ha comenzado a caracterizarse la española como una sociedad multicultural hasta que han aparecido y se han visibilizado los diversos colectivos de inmigrantes no comunitarios $^{1}$ en el territorio. Es decir, el discurso de la multiculturalidad se produce (o mejor, se reproduce, porque es de origen anglosajón), con la llegada de los extranjeros procedentes del denominado Tercer Mundo y se refiere a la diversidad representada por el colectivo identificado

\footnotetext{
${ }^{1}$ Una interesante reflexión sociológica sobre la categoría de "inmigrantes no comunitarios" puede consultarse en SANTAMARÍA, Enrique. La incógnita del extraño. Una aproximación a la significación sociológica de la "inmigración no comunitaria".
} 
como "inmigrante". Se olvida, por tanto, con excesiva frecuencia, que en el interior de no pocos países de la Unión Europea ha existido - y existen - grupos minoritarios que están detrás de esta (re)aparición de un nuevo modelo de sociedad multiétnica, que no procede sólo de los movimientos migratorios de terceros países, sino de las mismas sociedades en que vivimos que albergan en su seno esa pluralidad. ${ }^{2}$

La segunda observación que hay que tener en cuenta es que, una de las características del pluralismo cultural de nuestra sociedad, es la existencia una clara asimetría de poder entre diferentes grupos etnoculturales. Se trata de una asimetría vinculada a factores como el peso demográfico, el poder socioeconómico, las representaciones sociales de los grupos y la hegemonía cultural, además de la clara asimetría institucional relacionada con la nacionalidad comunitaria o no comunitaria. Cuando hablamos de integración es necesario tener en cuenta esta asimetría, para poder diseñar proyectos de intervención más eficaces en contextos multiculturales. Esta desigual distribución de poder es un factor que estructura la misma definición del grupo, puesto que existen grupos de personas que carecen incluso del poder de autodefinición identitaria.

La tercera observación sobre el término multiculturalismo es que, si bien la coexistencia de personas de diferentes horizontes culturales es un hecho, la convivencia, entendida como relación armoniosa e igualitaria sigue siendo un objetivo a alcanzar, en este sentido:

El multiculturalismo marcaría el estado, la situación de una sociedad plural desde el punto de vista de comunidades culturales con identidades diferenciadas. Mientras que la interculturalidad haría referencia a la dinámica que se da entre estas comunidades culturales. ${ }^{3}$

Desde este punto de vista, la multiculturalidad es un hecho objetivo en nuestra sociedad, independientemente de que ello se quiera o no; la interculturalidad, en cambio, es una respuesta ética, social y normativa (porque incluye ideales, valores y principios) a este hecho incuestionable. Una respuesta que implica importantes desafíos, entre los cuales repensar y superar los modelos hegemónicos de integración a la luz de un proyecto de ciudadanía universal que se fundamente en los Derechos Humanos.

\footnotetext{
${ }^{2}$ De LUCAS, Francisco Javier. “¿Elogio de Babel? Sobre las dificultades del Derecho frente al proyecto intercultural".

${ }^{3}$ RODRIGO ALSINA, Miquel. "Elementos para una comunicación intercultural", p. 13.
} 
El término integración es complejo, ambiguo y problemático, designa tanto un proceso de inserción en la sociedad receptora, como el resultado de este proceso. A menudo se limita a definir un "deber ser" voluntarista o se utiliza en el discurso político y en el discurso popular como eufemismo para definir los procesos asimilacionistas.

Integración es una palabra que salta con facilidad cuando
se habla de inmigración, está en la calle, en los medios de
comunicación y en los políticos. Quizá por ello ha dejado de
hacer referencia a un concepto concreto y se utiliza según la
idea que cada cual tiene de lo que debe ser la adaptación
de los inmigrantes a la sociedad receptora. Es un término
que, por tanto, usa todo el mundo y que parece darse por
sabido, pero del que en realidad se tienen ideas diferentes. ${ }^{4}$

La integración en el ámbito de las migraciones es un objetivo onmipresente, sin embargo sigue sin haber acuerdo respecto a qué se entiende por integración y cuáles son los medios para alcanzarla. Lo que se presenta habitualmente en los manuales, en los planes oficiales y en los ensayos no suele ser más que un modelo ideal normativo de integración, que no toma en cuenta aspectos contextuales que influyen en el proceso, como el hecho de que la inmigración sigue siendo, en muchos casos, un factor de desventaja social. Por otra parte, los procesos de integración se desarrollan generalmente en un contexto social (y discursivo) caracterizado por una retórica dominante basada en la asimilación subalterna y el racismo culturalista. El desarrollo de la convivencia intercultural pasa por un análisis crítico de estos dos modelos.

\section{La asimilación subalterna}

En el discurso mediático, popular y político a menudo se habla de integración para hacer referencia a procesos que son más bien de asimilación. El asimilacionismo es un modelo que privilegia la uniformización cultural: los grupos y las minorías étnicas, van a ir adoptando las normas, lengua, valores y señas de identidad de la sociedad mayoritaria con una paralela pérdida del propio horizonte cultural de origen. El mantenimiento de la cultura de origen de las minorías es considerado como una amenaza a la cohesión social y a la identidad cultural dominante. La asimilación implica una visión culturalista de la cultura de la sociedad receptora que es considerada como homogénea y

\footnotetext{
${ }^{4}$ PUMARES, Pablo, apud CHECA OLMOS, Juan Carlos; ARAGÓN BOMBÍN, Raimundo. Inmigración y derechos humanos: la integración como participación social, p. 294.
} 
sin fisuras. En este sentido, la homogeneidad es el punto de partida y es la meta del proceso de integración y se supone que la plena asimilación conllevaría la desaparición de los prejuicios y de la discriminación.

Es importante subrayar este aspecto porque es la base de un argumento muy común de la actual retórica racista: la culpabilización de la víctima. El asimilacionismo es una lógica de pensamiento que no sitúa los "problemas de convivencia o integración" en las condiciones de desigualdad vividas por los grupos minoritarios, sino en un supuesto "proceso de adaptación inacabado". Según esta visión, el racismo y la discriminación son causados por la falta de integración de determinados colectivos.

Hoy en día el término asimilación ha dejado de utilizarse, sin embargo, se trata en general de un simple cambio de fachada, puesto que el asimilacionismo sigue siendo el modelo dominante en el que se basan las políticas de integración y la opinión pública. Además no se trata de un modelo de asimilación "puro", puesto que en realidad no se favorece y no se permite la asimilación plena, sino que se propicia una "asimilación subalterna": la persona migrante puede quedarse siempre y cuando se la necesite y esté dispuesta a aceptar los trabajos rechazados por la población autóctona. Los hechos ocurridos en 2005 en Paris nos han enseñado que el resultado final de los procesos de asimilación no es la incorporación en la sociedad de acogida en igualdad de condiciones, sino la inclusión subalterna. Se aceptan a las personas migrantes siempre que no compitan con "los/as de aquí", siempre que no se casen con nuestros hijos o hijas y siempre que no vivan en nuestro barrio.

La asimilación subalterna se basa en el principio de "preferencia nacional" particularmente explotado por determinados partidos políticos en toda Europa: el lema "primeros los de aquí" se ha trasformado en un leitmotiv de las campañas electorales, es un principio que ha generado ecuaciones simplistas como: "iporque necesitamos a 4.000.000 de extranjeros cuando tenemos 4.000.000 de parados?".

Otro aspecto del discurso asimilacionista hace referencia a la responsabilidad única de las personas migrantes en el proceso de integración. En el último informe sobre la xenofobia en España ${ }^{5}$, Cea D’Ancona y Valles muestran cómo la frase "se deben adaptar al país donde residen" fue uno de los nodos discursivos reiterativos en el proyecto

${ }^{5}$ CEA D’ANCONA, María Ángeles; VALLES, Martínez. Evolución del racismo y de la xenofobia en España. Informe 2010, p. 81. 
MEXEES I. También lo fue en el proyecto Living Together: European Citizenship against Racism and Xenophobia, en el cual la demanda de integración-adaptación del inmigrante (entendida más en el sentido de asimilación de "ellos" a "nosotros") fluye en el discurso de la población adulta, tanto en España como en otros países participantes en el estudio: "Cuando estoy en el extranjero, me adapto a su cultura para que la gente pueda tolerarme" (Suecia); "Que vengan si no causan problemas y se adaptan un poco" (Holanda); "Los inmigrantes tienen que adaptarse completamente a la sociedad finlandesa" (Finlandia).

La sociedad considera a los inmigrantes sólo como mano de obra y no como personas con potencialidades y necesidades. Se pretende que los inmigrantes sean productivos pero que no participen en la sociedad, que estén al mismo tiempo dentro del sistema de producción y fuera del Estado de bienestar y de la vida pública. ${ }^{6}$

La metáfora de la "mano de obra barata" refleja el modelo implícito que está detrás de la política migratoria: el viejo modelo alemán del Gastarbeiter, la persona inmigrante como trabajador huésped, cuya presencia es justificable solamente porque es útil a la economía y desechable en momentos de crisis. Se legitima una imagen del inmigrante inferiorizada: el buen inmigrante es como el "Tío Tom", es humilde, gran trabajador, ha interiorizado su inferioridad, obedece y no reivindica derechos, es invisible fuera de su ambiente de trabajo.

\section{El racismo culturalista}

A partir del fin de la Segunda Guerra Mundial, los horrores del nazismo y las consecuencias de su ideología racista han desprestigiado toda manifestación, ideología o práctica discriminatoria relacionadas con el concepto de raza. En general podemos decir que, hoy en día, los estados democráticos se declaran abiertamente antirracistas y han creado un corpus de declaraciones (como por ejemplo la Carta de Derechos Humanos en 1948), leyes, decretos, etc. para prevenir y perseguir las prácticas discriminatorias. En términos generales las declaraciones abiertamente racistas son mal vistas y "ser racista" es, en la mayoría de los

\footnotetext{
${ }^{6}$ Es lo que resume la conocida y deplorable expresión del alcalde de El Ejido (Andalucía) con motivo de los primeros sucesos racistas allí acaecidos hace unos años: "A las siete de la mañana todos los inmigrantes son pocos, pero a las siete de la tarde sobran todos". Y lo que acertadamente denominó Javier de Lucas como "Modelo Blade Runner" de inmigración, en Le Monde Diplomatique (febrero, 2003).
} 
casos, estigmatizado socialmente. Sin embargo, esto no es incompatible con la existencia de un racismo institucional cada vez más legitimado socialmente, que se expresa tanto en los discursos de los representantes institucionales, como en la legislación de extranjería que recorta derechos, en las actuaciones de los funcionarios y empleados de servicios públicos y hasta en las políticas sociales que, supuestamente, se declaran no racistas.

Por lo tanto, esto no significa que el viejo racismo haya desaparecido, ni que el racismo este decayendo. Se han hecho importantes progresos, pero en el actual contexto social, más próximo al antirracismo que al racismo, se están desarrollando también nuevas formas de intolerancia que son más sutiles, menos explicitas. Las nuevas formas de racismo buscan su legitimación y "respetabilidad" a través de la supresión del término raza y de la utilización de términos con una connotación menos negativa: cultura, identidad y etnia. El resultado es un racismo "sin razas" que, además, usa el aparado conceptual propio de las propuestas multiculturalista y antirracista. Este universo conceptual se basa en la esencialización de la cultura y de la identidad cultural y se ha transformado, junto con el asimilacionismo subalterno, en la retórica dominante sobre la integración. Se reducen las variables que modulan el proceso de integración a los aspectos culturales: las culturas se transforman en entidades homogéneas y monolíticas, el concepto de cultura se racializa y se trasforma en atributo "casi genético" de las personas.

Las ideas dominantes sobre la cultura y la identidad europeas suelen estar arraigadas en una visión algo mística de la historia del continente europeo y ello conlleva una exclusión potencial del sentimiento de pertenecer plenamente a dicha sociedad de muchos ciudadanos no comunitarios, así como de muchos ciudadanos no comunitarios de un origen minoritario étnico o racial. ${ }^{7}$

El racismo culturalista ha alimentado una retórica hegemónica compartida, tanto por los partidos políticos xenófobos, como por exponentes del pensamiento liberal: es el principio de "iguales pero separados" y la inintegrabilidad de determinadas "grupos culturales".

Uno de los ejemplos más acabados de racismo culturalista "liberal" lo encontramos en el ensayo del politólogo italiano Giovanni Sartori, La sociedad multiétnica. Pluralismo, multiculturalismo y extranjero; donde argumenta que los inmigrantes musulmanes, por su religión-cultura

${ }_{7}$ MARTINIELLO, Marco. "Integración y diversidad en una Europa multicultural", p. 81. 
fundamentalista, son incompatibles con la sociedad democrática y con el pluralismo. Según el autor, el Islam amenaza los elementos básicos de nuestra identidad (democracia, libertad, igualdad, pluralismo, etc.). La consecuencia natural de esta argumentación es que los musulmanes no son integrables. En la base de esta lógica argumentativa de Sartori se encuentra la idea de que las culturas son homogéneas, inconmensurables e incomunicables. Según Sartori el Islam es por esencia fundamentalista, y consecuentemente incompatible con los valores propios de la sociedad democrática europea:

Toda comunidad implica clausura, un juntarse que es también un cerrarse hacia fuera, un excluir [...] la cultura asiática también es muy lejana a la occidental, pero sigue siendo "laica" en el sentido de que no se caracteriza por ningún fanatismo o militancia religiosa. En cambio, la cultura islámica sí lo es [...] Del mismo modo, la ley coránica no reconoce los derechos del hombre (de la persona) como derechos universales e inviolables, otro fundamento, añado, de la civilización liberal. Y éstas son las verdaderas dificultades del problema. El occidental no ve al islámico como un "infiel". Pero para el islámico el occidental sí lo es. [...] ¿̇hasta qué punto la tolerancia pluralista debe ceder no sólo ante "extranjeros culturales"? [...] La verdad banal es, entonces, que la integración se produce entre integrables y, por consiguiente, que la ciudadanía concedida a inmigrantes inintegrables no lleva a integración sino a desintegración. ${ }^{8}$

Las nuevas expresiones del racismo utilizan un argumento propio del discurso multiculturalista y antirracista para justificar la exclusión: el reconocimiento de la diferencia. En este caso, sin embargo, la diferencia entre culturas es concebida como un obstáculo insuperable para el dialogo y la integración. El reconocimiento de la diferencia, en este caso, no es el reconocimiento del otro, sino la definición de los otros grupos como desiguales y radicalmente diferentes. La lógica de la diferencia radical despoja a los otros de la posibilidad de hacer oír su propia voz, la diferencia es absoluta, el otro es mudo porque es incomprensible, no le oímos porque hemos decidido que no le podemos entender.

El filósofo Gabriel Bello, que ha realizado un lúcido análisis de la perspectiva de Giovanni Sartori, destaca:

\footnotetext{
${ }^{8}$ SARTORI, Giovanni. La sociedad multiétnica. Pluralismo, multiculturalismo y extranjeros, p. 48-53 y 114.
} 
Con la diferencia absoluta, tampoco se hace justicia a la diferencia. La diferencia absoluta que exige la inconmensurabilidad sólo se puede percibir en contraste con la diferencia no absoluta, la diferencia relativa, que implica una zona de semejanza o identidad igualmente relativas. Tanto la diferencia como la semejanza relativas sólo se perciben en un juicio de comparación entre dos o más elementos (por ejemplo culturas) que exige la presencia de un término de comparación o término medio. ${ }^{9}$

Una de las principales implicaciones de la construcción de la "diferencia absoluta" entre nosotros y ellos es la idea de que existan algunas culturas incompatibles con los valores democráticos y personas no integrables. Resulta claro que se trata de una nueva expresión de la lógica del racismo: las culturas como un todo homogéneo, perfectamente separadas entre ellas, independientes, estables. La cultura tiene las mismas características de la raza, es algo genético, no modificable. En esta perspectiva una persona de origen extranjero, aunque tenga la nacionalidad del país de acogida, siempre será extranjera. Como declaraba de Enoch Powell en Estrasburgo en noviembre de 1968: "Un antillano o un asiático no se convirtió en inglés por el hecho de nacer en Inglaterra. Es ciudadano británico ante la ley, por su nacimiento, pero en realidad sigue siendo un antillano o un asiático"10.

Resumiendo, el discurso dominante sobre la integración hoy día es asimilacionista y culturalista. Se perciben determinadas diferencias como absolutas y radicales y como desviación de la norma. Se exacerban y exageran las diferencias culturales en particular respecto a determinados colectivos, como "los musulmanes" o "los árabes". Se considera que existen colectivos cuya "distancia cultural" es tan grande que no son integrables. La cohesión social es concebida como homogeneidad cultural y las personas inmigrantes se aceptan siempre que acepten nuestras costumbres sin poner en discusión las relaciones asimétricas de poder.

\section{Más allá de la estética intercultural}

Frente a las derivas racistas del discurso dominante sobre la integración y la convivencia en contextos multiculturales se ha desarrollado un modelo de gestión de la diversidad, particularmente presente en el discurso y en las prácticas educativas y sociales, que podríamos definir

\footnotetext{
${ }^{9}$ BELLO, Gabriel. El valor de los otros. Más allá de la violencia intercultural, p. 80.

${ }^{10}$ Apud WIEWIORKA, Michel. El racismo. Una introducción, p. 41.
} 
como "estética intercultural". Se trata de un modelo que pone el acento en la interacción entre "culturas", en la tolerancia y en el respeto y las oportunidades positivas que abre la diversidad cultural. Sin embargo la interacción es pensada de forma superficial e ingenua, reduciéndola en una celebración de una "estética intercultural", sin tener en cuenta la naturaleza dinámica, fluida y flexible de las culturas y la complejidad de las relaciones de dominación existentes en los espacios multiculturales.

Más allá de las buenas intenciones, este modelo tiene algunas características que pueden obstaculizar la convivencia intercultural. La primera es la folclorización de las diferencias, se trata de un culturalismo edulcorado, una visión de las culturas "de escaparate". Se corre el riesgo de reproducir una visión reducida y estática de las culturas y encerrar las personas en categorías estereotipadas más cercanas a las guías turísticas que a su realidad personal.

La segunda característica es la invisibilización de las relaciones asimétricas de poder y de la desigualdad de derechos. Lo que conlleva inevitablemente a la ilusión que la convivencia intercultural pueda ser el fruto, simplemente, de las buenas intenciones. Los festivales "interculturales", las comidas "interétnicas", las proclamas de tolerancia y la celebración de la diferencia sirven de poco si no se reconocen los derechos básicos, si no se lucha contra la asimetría de poder y si no se empieza con la igualdad de condiciones sociales.

\section{Los desafíos de la integración intercultural}

La superación de las tres perspectivas anteriormente presentadas: el asimilacionismo subalterno, el racismo culturalista y la estética intercultural pasa por el desarrollo de un enfoque intercultural crítico y trasformador. No se trata sólo de aceptar y respetar las diferencias y de valorizar la interacción entre personas de diferentes culturas, sino de poner en discusión la visión estática y esencialista de la cultura y de la identidad, así como de plantear una nueva ética de la convivencia y un nuevo ideario político.

El interculturalismo tiene una visión dinámica y abierta de las culturas, se basa en un proceso de diálogo, negociación y aprendizaje mutuo, tomando conciencia del pasado colonial y del presente imperialista de nuestro horizonte cultural.

En este contexto podemos definir la integración como: 
Un proceso de adaptación mutua y de creación de una nueva ciudadanía, entendiéndola como una praxis generadora de interacción positiva entre personas, grupos o instituciones de culturas diversas, a partir de la búsqueda y promoción de relaciones de igualdad, respeto a la diferencia y convivencia social. ${ }^{11}$

Los principios que deben presidir la aplicación práctica de la convivencia han de ser la igualdad de derechos, condiciones, obligaciones y oportunidades con la población autóctona, así como el principio de la igualdad de culturas y el derecho a la propia identidad; se deben combinar los planteamientos socioeconómicos y los legales con los culturales y se debe trabajar en un marco de interculturalidad porque significa interrelación, dinamismo y adecuaciones mutuas. De este modo, la interculturalidad aleja los peligros de guetización que se pueden dar en algunas interpretaciones multiculturalistas, sin tener que renunciar al derecho a la propia identidad lingüística, religiosa o cultural. Desde esta concepción intercultural, no se parte de la concepción del inmigrante como un sujeto culturalmente diferente sin más, sino desde su categoría de sujeto de derechos, inscribiendo su derecho a la diversidad en el marco más amplio de la igualdad general de derechos.

Como hemos subrayado anteriormente el enfoque intercultural no se limita a reconocer y valorizar las diferencias, sino que subraya la importancia de la convivencia basada en la comprensión recíproca y el diálogo intercultural. Éstos, sin embargo, no son algo "natural" y "automático". A menudo no tenemos a nuestra disposición los repertorios interpretativos para comprender la complejidad social y la diversidad cultural de nuestra sociedad. No es suficiente con incrementar el contacto y las ocasiones de diálogo para que las personas se comprendan más y mejor: el contacto puede alejar las personas en lugar que acercarlas. El interculturalismo implica el desarrollo de nuevas competencias que nos permiten relacionarnos eficazmente con personas que no comparten nuestro horizonte cultural. En este sentido la convivencia intercultural es un proceso bidireccional y no unilateral. Ambas partes implicadas, tanto la minoría como la mayoría, interactúan, negocian y generan espacios de participación y de identificación mutua que trasforman a todos los actores participes, no únicamente al grupo minoritario. "Un proceso integrativo nunca puede ser unilateral, ya que se basa en el

\footnotetext{
${ }^{11}$ GIMÉNEZ, Carlos (dir.). Dotación de instrumentos de intervención para la inserción laboral de inmigrantes y refugiados: Análisis de la realidad y formación de profesionales en mediación laboral, p. 186.
} 
encuentro, en la comunicación y en el intercambio; no puede decretarse unilateralmente, sino que es constantemente negociado"12.

La bidireccionalidad del proceso de integración y de convivencia tiene dos importantes implicaciones a la hora de planificar las estrategias de intervención social con personas inmigrantes: por un lado amplía el ámbito de actuación de los proyectos incluyendo toda la sociedad receptora, por el otro valoriza el papel activo de las personas migrantes como coprotagonistas del proyecto de integración. El punto de partida no es solamente el análisis de los problemas, sino las competencias de las personas migrantes, sus redes, su capital social y cultural, su resiliencia. El análisis de la realidad y de las prioridades de intervención tiene que centrarse en la persona, en sus circunstancias y sus experiencias. Se trata de valorizar su resiliencia, su capacidad de enfrentarse a las adversidades y de salir adelante, se trata de tomar en consideración su historia previa, sus prácticas, sus redes de apoyo, centrarse en las capacidades y no en las carencias, en las fortalezas y no en las debilidades, en las posibilidades más que en las limitaciones.

El proceso de adaptación recíproca está condicionado por factores coyunturales y situacionales que tienen una gran importancia a la hora de comprender la integración. Como hemos visto el discurso dominante se centra en los factores culturales, olvidando que la situación administrativa, "el tener o no tener papeles", es una de las variables que más influye en los procesos de integración. A este propósito es lícito preguntarse si no existe una clara contradicción entre las políticas de integración europeas y españolas y las políticas que regulan los flujos migratorios. Son éstas últimas las que construyen la "ilegalidad" y legitiman la creencia social acerca de la existencia de los "buenos" (los regulares) y "malos" inmigrantes (los que están en situación administrativa irregular). iSe pueden exigir unos deberes como ciudadano/a y una integración social plena a quien no tiene los derechos básicos reconocidos?

La primera condición sin la que no puede haber integración es el reconocimiento de derechos en igualdad con la población autóctona. No sólo derechos sociales básicos (como educación, sanidad o empleo); sino también los derechos de ciudadanía, de participación política, que potencian la integración política, el sentimiento de pertenencia al espacio donde se habita. Pero esto último no siempre se reconoce. El énfasis suele recaer más bien

${ }^{12}$ GARCÍA ROCA, Javier. “Integración”, p. 203-211. 
en las dimensiones sociales y culturales de la integración, relegándose la dimensión política. ${ }^{13}$

Por esta razón una política de integración eficaz tiene que desarrollarse en la triple dimensión de las llamadas " $3 R^{\prime \prime 14}$ : las políticas de "redistribución" que hacen referencia a la igualdad social; las políticas de "reconocimiento" de la diferencia cultural o identitaria; y las políticas de "representación" que implican los derechos políticos y de ciudadanía.

Por lo tanto, la premisa fundamental para la integración social del inmigrante se basa en su reconocimiento como ciudadano, con derechos y deberes, más que en su identidad. Como tan acertadamente ha señalado en numerosos trabajos Javier de Lucas, los flujos migratorios tienen un carácter radicalmente político. Se trata de un fenómeno global, complejo y plural que debemos tomar ya en serio como una de las cuestiones políticas clave, que exige paciencia y visión a medio y largo plazo, siendo el reconocimiento de derechos una condición previa y necesaria (aunque no suficiente) para que haya una política y una realidad social de integración. ${ }^{15}$ La integración es un proceso enormemente complejo, en el que intervienen factores psicológicos, sociológicos, políticos, económicos y culturales. Pero, sin el reconocimiento previo de derechos y su posibilidad de ejercicio, sólo cabe hablar de políticas de integración parcial. Integrar es, primeramente, equiparar en derechos y todo lo que sean reformas legislativas que recortan derechos de las personas inmigrantes son actuaciones en contra de su integración social. Hay que procurar, por tanto, una amplia equiparación de derechos desde el principio y no demorar el momento en el que la persona inmigrante pueda tener exactamente los mismos derechos que el resto de la población (incluido el derecho al voto), que bien podría coincidir con el momento de la obtención de la residencia permanente. Del mismo modo, la integración se ve dificultada seriamente por el sistema de cierre de fronteras, que condena a un porcentaje elevado de personas a pasar por un período más o menos largo de irregularidad, retrasando notablemente su proceso de integración social.

Existen grandes diferencias en el proceso de integración relacionadas con la situación económica, laboral y administrativa de las personas

\footnotetext{
${ }^{13}$ CEA D'ANCONA, VALLES, op. cit.

${ }^{14}$ CACHÓN, Lorenzo. La España inmigrante: marco discriminatorio, mercado del trabajo y políticas de integración.

${ }^{15}$ De LUCAS, Francisco Javier. "Los nuevos movimientos migratorios en el siglo XXI. Otras miradas. Otras respuestas".
} 
inmigrantes, de modo que, a mejor nivel económico alcanzado, mejor es el proceso de adaptación y participación, y viceversa. El verdadero proceso de adaptación sólo puede iniciarse cuando los individuos hayan resuelto sus necesidades vitales básicas, como lo son el trabajo, la vivienda, la educación, la salud, etc. La inseguridad laboral, la falta de vivienda digna y el hacinamiento o la cohabitación forzada (vivir varias familias en la misma vivienda), producen grandes dificultades en el proceso de integración y participación, generan estrés, ansiedad, fatiga emocional y, en ocasiones, episodios de agresividad, debido, sobre todo, a la sensación de inseguridad.

Finalmente, la integración intercultural implica una ampliación de los espacios de participación social.

Una sociedad integrada es aquélla que abre espacios para la participación, para la deliberación, para el debate de opiniones, para la creación de una opinión pública. La integración no consiste en entrar en un sistema cerrado, sino que se produce activamente en el encuentro; no consiste en incorporarse a un nuevo sistema sino la apuesta. ${ }^{16}$

Por un lado, se trata de ampliar y de crear nuevos canales de participación, por otro, de integrar el concepto mismo de participación en un contexto que supera el espacio nacional. Muchas personas migrantes no participan solamente en la sociedad receptora, sino en un campo migratorio cuyos polos son la sociedad de origen y la sociedad receptora. Viven en un espacio transnacional, a través de sus remesas económicas y sociales se hacen presentes en su comunidad de origen. El impacto de este conjunto de múltiples actividades ha de ser tenido en cuenta, tanto en los contextos de salida, como en los de llegada y tránsito de los migrantes.

\section{Conclusiones}

La diversidad cultural constituye un asunto central y de importancia cada vez más creciente en la dinámica y evolución de las sociedades actuales. La multiculturalidad, por tanto, es una característica incuestionable y cada vez más presente en las mismas.

La gestión de este hecho, sin embargo, se realiza desde perspectivas y respuestas muy variadas y, no pocas veces, de dudosa eficacia en el

\footnotetext{
${ }^{16}$ MELERO VALDÉS, Luisa (coord.). La persona más allá de la migración. Manual de intervención psicosocial con personas migrantes, p. 27.
} 
terreno de la convivencia ciudadana para una ciudadanía inclusiva. La respuesta de quienes proponen un "intervencionismo negativo", que elimine y expulse a los individuos considerados extraños, no ha sido tratada en este artículo porque tal respuesta es absolutamente condenable desde el punto de vista de la ética convivencial y los Derechos Humanos. Ahora bien, aceptada la necesidad de construir la convivencia cívica en una sociedad multicultural, nadie puede negar el hecho de que su adecuada gestión conlleva importantes desafíos, a los que no se está dando, con frecuencia, la respuesta racionalmente más adecuada.

Hemos analizado las tres respuestas más comunes a este desafío, que hemos denominado y caracterizado como "asimilación subalterna", "racismo culturalista" y "estética intercultural". Asimismo, se han identificado algunas de las limitaciones, carencias y problemas que estas respuestas evidencian, y que imposibilitan y dificultan en muchos casos el objetivo que supuestamente persiguen, como es el de contribuir a la construcción de una ciudadanía inclusiva y su correlato práctico de una convivencia intercultural transformadora y superadora de las desigualdades y asimetrías de poder entre grupos sociales.

Frente a estas respuestas comunes y sus falencias, hemos destacado la necesidad de desarrollar una respuesta interculturalista crítica y transformadora, que consideramos una alternativa válida para superar esas barreras, desigualdades y asimetrías que subyacen en las tres respuestas analizadas previamente. Por su importancia e implicaciones, apostamos por el desarrollo de un interculturalismo crítico y transformador. El enfoque intercultural que hemos propuesto es crítico porque implica la deconstrucción del asimilacionismo subalterno y del racismo culturalista además de la superación de ciertas formas reduccionistas de entender el interculturalismo; y es transformador porque supone repensar nuestra forma de concebir la identidad, la cultura, la participación y la ciudadanía.

El interculturalismo es una respuesta normativa al hecho de la pluralidad cultural, y se basa en una efectiva convivencia, aprendizaje y enriquecimiento mutuos. Este enfoque se diferencia y opone a otras respuestas normativas tales como la "asimilación" de las culturas minoritarias por parte de la cultura mayoritaria y se opone también a la mera "tolerancia mutua" del "iguales pero separados".

Nuestro enfoque crítico-transformador del interculturalismo no se limita al reconocimiento de la diferencia y a la promoción de la interacción 
positiva entre personas o grupos con diferentes horizontes culturales de referencia, sino que apuesta por la lucha contra las desigualdades sociales, económicas y políticas; y contra las discriminaciones étnicas, raciales y culturales.

\section{Bibliografia}

BELLO, Gabriel. El valor de los otros. Más allá de la violencia intercultural. Madrid: Biblioteca Nueva, 2006.

CACHÓN, Lorenzo. La España inmigrante: marco discriminatorio, mercado del trabajo y políticas de integración. Barcelona: Anthropos, 2009.

CEA D'ANCONA, María Ángeles; VALLES, Martínez. Evolución del racismo y de la xenofobia en España. Informe 2010. Madrid: Ministerio de Trabajo e Inmigración, 2010.

De LUCAS, Francisco Javier. "¿Elogio de Babel? Sobre las dificultades del Derecho frente al proyecto intercultural", in NAÏR, Samy; De LUCAS, Francisco. EI desplazamiento en el mundo. Inmigración y temáticas de identidad. Madrid: IMSERSO, 1998.

. "Los nuevos movimientos migratorios en el siglo XXI. Otras miradas. Otras respuestas", in Revista de Treball Social, v. 173, 2004, p. 41-63.

GARCÍA ROCA, Javier. "Integración", in CONILL, Jesús (coord.). Glosario para una sociedad intercultural. Valencia: Bancaja, 2002.

GIMÉNEZ, Carlos (dir.). Dotación de instrumentos de intervención para la inserción laboral de inmigrantes y refugiados. Madrid: Programa Migración y Multiculturalidad de la UAM, 2003.

MARTINIELLO, Marco. "Integración y diversidad en una Europa multicultural", in Vanguardia, Dossier marzo 2007, v. 22, 2007, p. 78-83.

MELERO VALDÉS, Luisa (coord.). La persona más allá de la migración. Manual de intervención psicosocial con personas migrantes. Valencia, Ceimigra, 2007.

PUMARES, Pablo, apud CHECA OLMOS, Juan Carlos; ARAGÓN BOMBíN, Raimundo. Inmigración y derechos humanos: la integración como participación social. Madrid: Icaria, 2004.

RODRIGO ALSINA, Miquel. "Elementos para una comunicación intercultural", in Revista CIDOB d'Afers Internacionals, v. 36, 1997, p. 11-21.

SANTAMARÍA, Enrique. La incógnita del extraño. Una aproximación a la significación sociológica de la "inmigración no comunitaria". Barcelona: Anthropos, 2002.

SARTORI, Giovanni. La sociedad multiétnica. Pluralismo, multiculturalismo y extranjeros. Madrid: Taurus, 2001.

WIEWIORKA, Michel. El racismo. Una introducción. Barcelona: Gedisa, 2009. 


\section{Abstract}

\section{The challenge of intercultural coexistence}

Cultural diversity is a central issue with ever-increasing importance in the dynamics and evolution of current societies. However its management is done from very different perspectives and responses, not infrequently, of dubious effectiveness in the field of civic coexistence for an inclusive citizenship. In this article the three most common responses to this challenge are analyzed, which have been named and characterized as "subordinate assimilation", "culturalist racism" and "intercultural esthetics". This way, some limitations, shortcomings and problems highlighted by these challenges were identified. In this context, there is the need of developing an intercultural, critical and transformative response, which consists in a valid alternative for the development of a coexistence based on dialogue, equality and recognition of differences.

Keywords: Interculturalism; Coexistence; Assimilation; Integration; Culturalist racism.

Recibido para publicación en 30/03/2012. Aceptado para publicación en 03/05/2012. Received for publication on March, 30 ${ }^{\text {th }}, 2012$. Accepted for publication on May, 03 ${ }^{\text {th }}, 2012$. 